\title{
Load Cells in Force Sensing Analysis - Theory and a Novel Application
}

\author{
Ivan Muller, Renato Machado de Brito, Carlos Eduardo Pereira, \\ and Valner Brusamarello
}

oad cells have long been used to sense and measure force and torque. When properly designed and used, they are very accurate and reliable sensors. Load cells are applied in several different fields, usually for weighing measurements. Among many other things, food, vehicles, and animals are weighed daily with load cells. The gripper of a robotic arm that picks up an object can be equipped with load cells in order to provide compression force feedback to the control system to prevent the object from being damaged or released too early. Also, load cells can be used to sense the compression forces during a robot's walk to provide data for the equilibrium-controlling system. In industrial machinery, rods, beams, wheels and bars are instrumented in order to check the forces exerted on them. The volume or level of a tank can be measured indirectly by means of a load cell that monitors the total weight. Lift units can also have a load's total weight measured to prevent overload. Because of such a variety of possible applications, load cells are very important.

This paper describes some of the theory and practice of load cells, including their basic elements and the electronics necessary for measurement. As an application example, the development of a 3-ring spherical load cell is presented which can be used to measure compression forces on fruit during storage and transportation is presented.

\section{A Ring-type Load Cell}

Aload cell is a device which contains other components. Aload cell is a device which contains many components. The most widely used is the strain gauge which is a thin foil resistor, the primary sensing element. They are bonded on elastic materials, such as metals, and have a specific geometry designed for a specific application. The strain gauge resistance changes according to the deformation of the spring element. What is strain? Strain is the deformation that occurs in a material when it is submitted to a stress. Stress is force acting on a specific area of a material. When stress is applied to conductors, there is variation in electrical resistance. This phenomenon was discovered by Lord Kelvin in 1856.

\section{The Strain Gauge}

When an object is subjected to mechanical stress, it contracts or expands. This is called the Poisson effect to honor the French scientist Simeon Poisson. Spring elements that exhibit Poisson effects are used with the addition of strain gauges that vary in electrical resistance when under stress. To measure the stress, strain gauges are attached to the spring element surface that contracts or expands and the mechanical changes in the surface are transmitted to the gauge. The intensity of the electrical resistance variation in the strain gauges is proportional to the intensity of the applied force. Figure 1 shows the basic ringtype geometry of a load cell. The four wide-black rectangles represent the placement of the strain gauges. Compared with the older devices, the actual strain gauges have evolved and can be considered vector strain sensors. They are able to indicate force, intensity, direction, and the method of applying the mechanical force.

\section{The Spring Element}

The spring element is connected to the object applying the force. When an external tension is applied, the spring element shape deforms/bends. Each type of spring element has a longitudinal (along the $\mathrm{x}$-axis) and a transversal (along the y-axis) Poisson effect according to its material composition. A spring element is usually made of aluminum or steel and the force applied to it must be within the elastic range of the material where it responds linearly. If it is overloaded beyond the elastic limit, it can suffer permanent damage. Figure 2 shows a metal beam with length $x$, width $y$, that is submitted to a strain $\varepsilon$ when a traction force is applied.

The ratio of the dimension variations is called the Poisson ratio, denoted by $v$. It is obtained by:

$$
\begin{aligned}
& \varepsilon_{l}=\frac{\Delta x}{x} \\
& \varepsilon_{t}=\frac{\Delta y}{y} \\
& \varepsilon_{t}=-v \varepsilon_{l}
\end{aligned}
$$




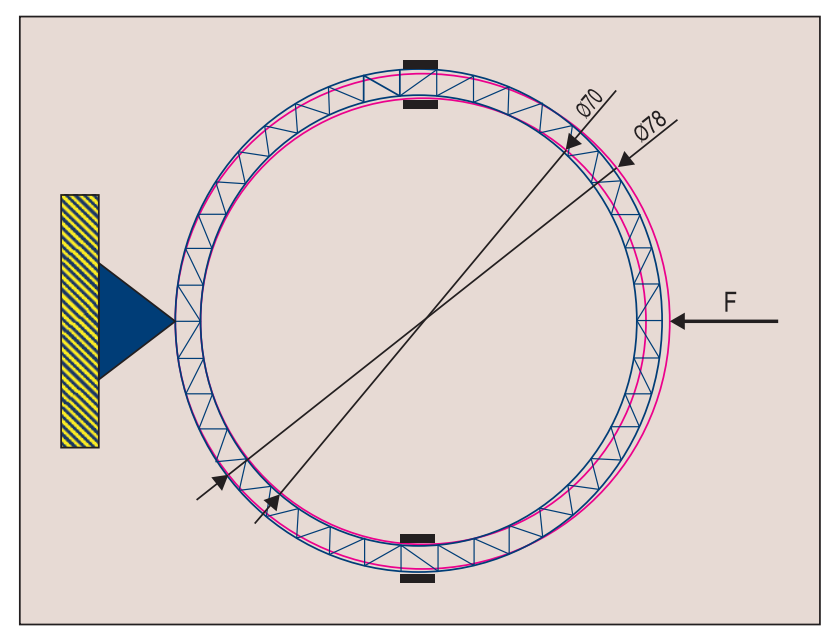

Fig 1. Drawing of a ring type load cell. The black marks (orthogonal to the force) indicate the strain gauge placements. (C) IEEE, Proc. IIMTC 2008, used with permission), [1].

where $\varepsilon_{1}$ and $\varepsilon_{\mathrm{t}}$ are the longitudinal and transversal strain, respectively. The Poisson ratio is $-\varepsilon_{1} / \varepsilon_{\mathrm{t}}$. This ratio varies from 0 to 0.5 for most materials. The rare auxetic materials are exceptions; they have a negative Poisson ratio.

The electrical resistance of a conductor (in our case, the strain gauge) is a function of its length and cross-sectional area. Therefore, if its length increases and its area decreases, the resistance will increase. This is established by the well known Ohm's law for resistivity, $R$ :

$$
R=\rho \frac{l}{A}
$$

where $\rho$ is the conductor's resistivity $(\Omega \cdot \mathrm{m}), l$ its length $(\mathrm{m})$, and $A$, the cross-sectional area $\left(\mathrm{m}^{2}\right)$. The strain gauge-spring element has an electrical resistance $\mathrm{R}$. The parameters are combined and renamed as the gage factor [2] that is used to quantify strain gauges:

$$
\text { Gauge }=\frac{\partial R / R}{\partial L / L}=1+2 v+\frac{\partial \rho / \rho}{\partial L / L}
$$

The bending magnitude on the spring element is small and normally expressed as microstrain by strain, $\mu \varepsilon / \varepsilon$. As a consequence of these small dimensional variations, the strain gauges generate small resistance variations, from 0.1 to 0.3 $\%$ of its nominal value. For a typical $120 \Omega$ strain gauge, the maximum value would be $120.36 \Omega$.

These small variations are one of the main problems of strain gauges. Other problems are related to their unwanted resistance variations due to temperature. In order to minimize these effects, Wheatstone bridges are used to measure electrical resistance and impedance with very small variations [3]. The measurement is indirect by the detection of the differential voltage in the center of the bridge. The circuit actually converts resistance variation into voltage variation and eliminates the offset voltage that naturally appears in a voltage divider, a simple resistance-to-voltage converter.

A Wheatstone bridge is formed by using 1, 2, or 4 strain gauges, then adding an amplifier and analog filters for

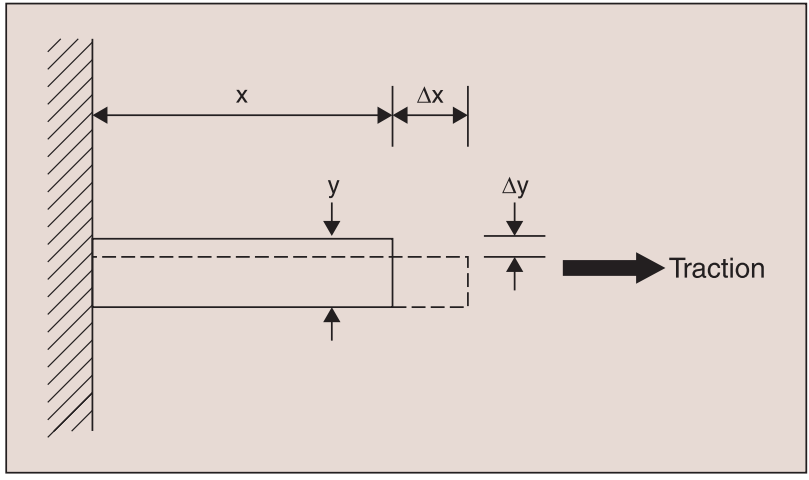

Fig 2. Beam element submitted to a traction force.

conditioning. Unfortunately, Wheatstone bridge strain gauges are not only sensitive to mechanical stress but also to heat variations, thermal instability [4], and noise. There is also the unbalance produced by the extra resistance from the wire connections. Several different techniques can be employed in order to minimize these problems. Basically, we have found that a full bridge load cell produces the most sensitive outputs with the best external effects cancellation, and it is usually chosen when there is enough space. Another way to maximize bridge sensitivity is to use higher excitation voltage. This is a trade off because of the self heating that then occurs in the strain gauges.

\section{Signal Conditioning}

The basic signal conditioning of the data from the strain gauge includes amplification and excitation of the analog signal from the load cell data, filtering, AD modulation, demodulation, and dynamic compensation.

- Amplification of the voltage signal from the Wheatstone bridge is necessary. Usually, a strain gauge Wheatstone bridge has $2 \mathrm{mV} / \mathrm{V}$ of sensitivity. With the typical $3 \mathrm{~V}$ of cell excitation, only $6 \mathrm{mV}$ will be available at full scale, and it requires high amplification. An instrumentation amplifier is needed with high input impedance, high differential gain, high common-mode and power supply rejection ratio, low drift, low offset and low input bias currents [5]. Meeting these requirements usually results in low speed performance, but that is not an issue in the weighing system's static measurements. Integrated instrumentation amplifiers can be used since they have the advantage of internal trimmed resistors for a better match and increase in overall performance.

D Filtering prevents aliasing and removes noise outside the signal's useful band. Noise is introduced in load cell systems by external or internal electromagnetic sources. The most common is the power line frequency that can be rejected easily by a low pass filter. The low pass filter, with low cutoff frequency, is usually employed because of the near static force measurement results. If dynamic force measurements are needed, things become more complex. Different techniques such as modulation and synchronous detection can 
be employed. Of course, we should never forget good shielding practices.

- Analog signal conditioning is being gradually reduced and substituted with digital conditioning, especially at the linearization process. In the digital world, there is no drift, component tolerance or aging. But the signals will always be analog, and thus, the front end (amplification and filtering) will be also. Ideally, all the ADC dynamic range should be used to take advantage of its full resolution. To do this, a low noise instrumentation amplifying and filtering are needed.

D After the correct conditioning, the signal is ready to be digitized. Force measurement demands low rate, low noise and high resolution quantification. For most applications of non-dynamic measurements, the sampling time is at long intervals. To diminish noise, averages of four or more acquisitions are performed before data are used. This can be done by multi-slope or sigma-delta ADCs. Resolutions greater than 16 bits and sample rates from 10-1000 S/s are quite adequate. In this work, we used a $1 \mathrm{kS} / \mathrm{s}$ sigma-delta ADC and $100 \mathrm{~Hz}$ low pass filtering (RC and sample averaging). Today, there are many ADCs that meet these requirements. There are also Systems-on-a-chip (SoC) available that includes a microcontroller, high resolution $\mathrm{AD}$, programmable gain amplifiers, filtering and communication peripherals.

\section{Other Load Cell Designs}

There are several different types and shapes of strain gauge based load cells, including shear beams, bending beams, buttons, rings and canisters. They are capable of sensing compression and traction forces and can be used directly or indirectly in several different applications. For multidimensional, there are some commercial load cells able to capture forces along one axis by means of a central rod. The rod is equipped with strategically positioned strain gauges in order to acquire not only compression and traction but also the axes moments. Some types are more sophisticated and capture the three axes forces and their angular moments with a total of six components.

The work presented in [6] is a multiaxial load cell composed of discs linked by rods. Again, the rods are equipped to sense strain. The compression and moment forces of each axis are obtained by means of extensive instrumentation that add and subtract the strain gauge signals. This ingenious device is capable of supplying extensive force information. To do this, all the load cell signals must be amplified accurately in order to provide low error force values. This can be quite laborious considering drift, offset and aging of the signal conditioning circuits.

If only one compression axis is needed, a simple load cell like a shear beam can be used to do the job. If two or three axes are needed, there are two possibilities: make use of a complex load cell like those described above or make use of a combination of simple load cells as we explain in the next section.

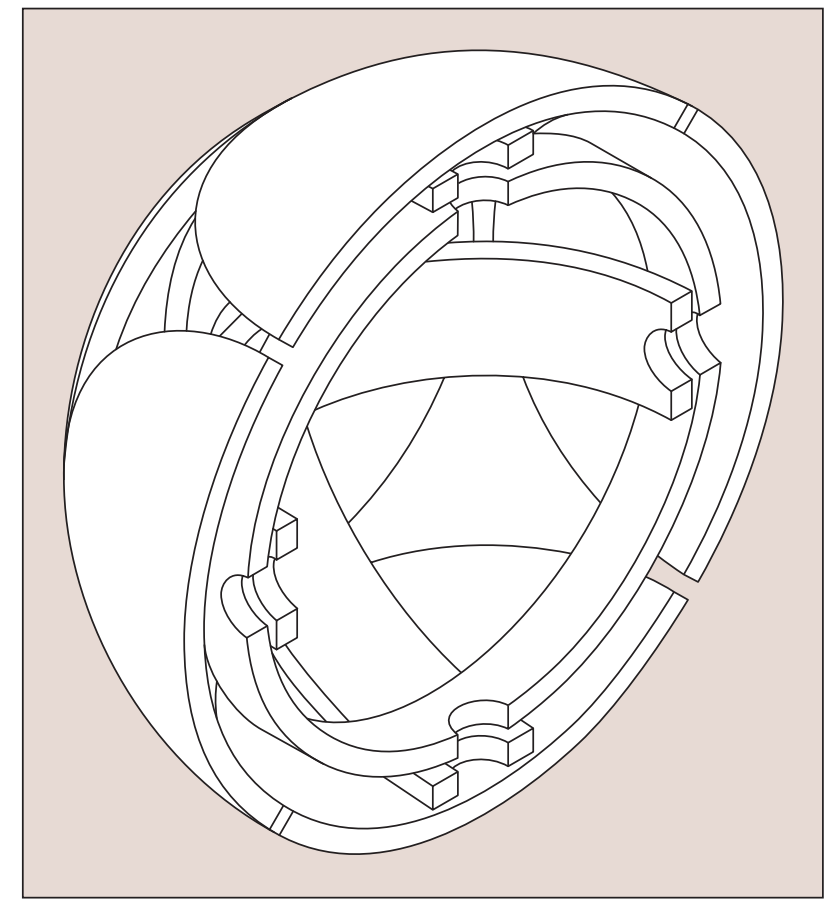

Fig. 3. Drawing of a final prototype's cut perspective. (C) IEEE, Proc. IIMTC 2008, used with permission), [1].

\section{A New Sphere-shape Load Cell Design}

We have developed several different load cells to sense all of the force components that act at only one point. If the forces come from different directions, a combination of load cells can be used. If only three axis compression/traction forces need to be found, we propose using a different load cell configuration. The device is composed of a combination of 3-ring load cells which simplifies the design. Compression and traction forces that are totally independent for each axis are measured, but moment forces are not. This load cell was used to quantify compression and impact forces suffered by fruits during the post-harvest and transportation process [1]. The first prototype was built with a diameter of $80 \mathrm{~mm}$, which is the approximate diameter of an ordinary apple. The dimension and positioning of the rings were determined with the help of a stress calculation software which used a finite element decomposition analysis. The rings' thickness were designed to allow a maximum of $500 \mathrm{~N}$ on each ring during an experiment. This force was considered to be adequate for the application (at that time, the real values of compression forces experienced by the fruit were not known. Rings were so designed so that maximum bending and independence and uncoupling of forces were possible).

Figure 3 shows the mechanical assembly. The three different sized rings are inside each other. There are rods and screws to keep them together while still allowing them to bend according to one of the three axes. In this way, the rings cannot rotate or slide. There are covers on the rings like hub caps to form a sphere-shaped volume and to concentrate the force on only one point. Actually, the rings are compressed, so there is an associated area with the force $\left(\mathrm{N} / \mathrm{m}^{2}\right)$. 


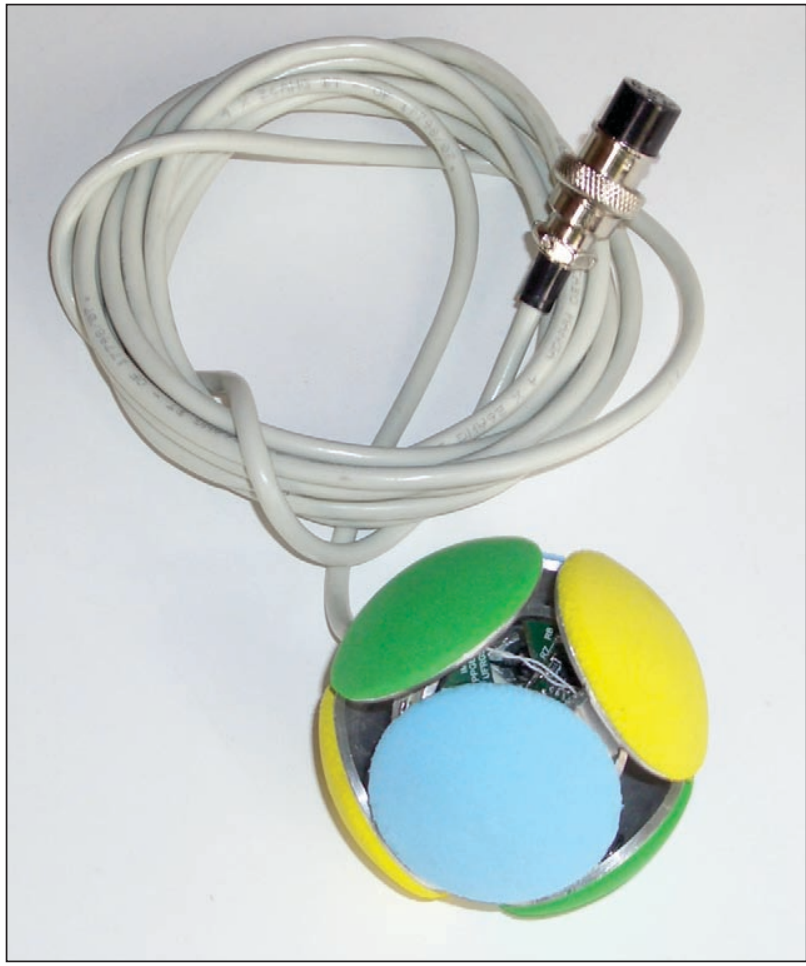

Fig. 4. Sphere shaped load cell.

Four strain gauges are attached to each ring to comprise a Wheatstone bridge and the generated signal is conditioned by an instrumentation amplifier and filtered before the ADC. The ring load cells were chosen because of their low cost, simplicity and reliability, but it was difficult to assemble them to form a sphere-shaped load cell. Figure 4 shows one of the assembled prototypes. The correct strain gauge's position is orthogonal to the force axis, which corresponds to the position where the maximum bending occurs.

Attachment of strain gauges in a ring spring element may cause two main problems: the generation of an offset by the Wheatstone bridge imbalance due to its internal and external curvature and the angular misalignment. The error due to bad angular positioning can be avoided, but the imbalance due to curvature cannot. Because of this, all the developed prototypes presented an initial imbalance that had to be corrected by an auxiliary parallel trimming resistance.

The signals from the Wheatstone bridge of each ring load cell are amplified by instrumentation amplifiers. The initial gain value was estimated in terms of the maximum compression force chosen for the load cell, $500 \mathrm{~N}$ during an experimental procedure when the rings were compressed with a hydraulic press in series using a pre-calibrated load cell. With the correct amplification, the corresponding output voltage of each ring load cell was digitized. This allowed us to exploit almost all of the dynamic range of the ADC. The voltage excitation of the Wheatstone bridge is asymmetrical because the load cells only sense compression, not traction, and the bridge always has a positive imbalance. As the sphere weighs itself, the remaining offset must be compensated. The software subtracts the extra value. This approach permits corrections

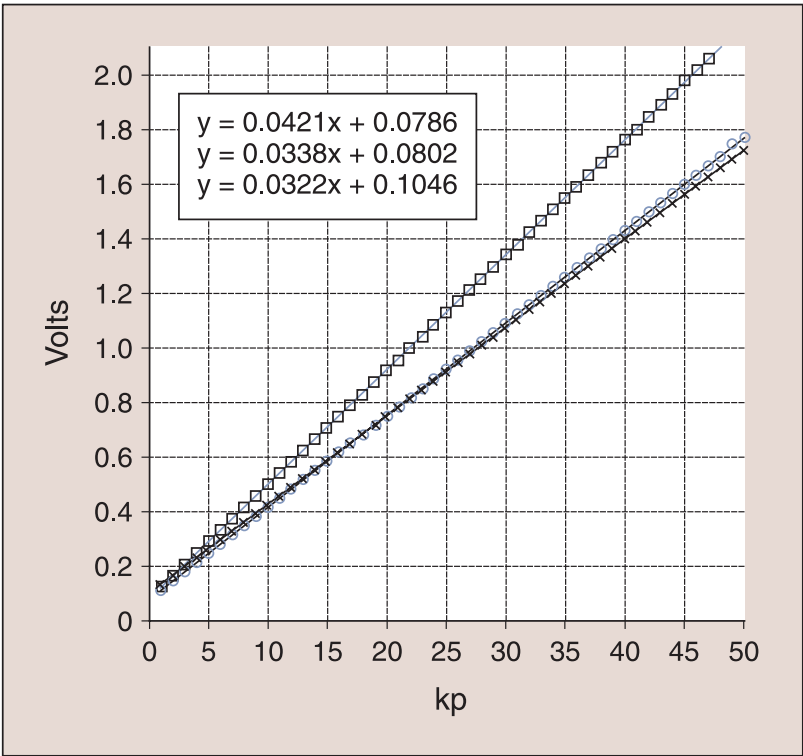

Fig. 5. Calibration results for each ring of the load cell. (C) IEEE, Proc. IIMTC 2008, used with permission), [1].

during the use of the sphere because the offset changes every time the sphere is repositioned.

To keep the bridge circuit consumption as low as possible, the excitation voltage was reduced to $1.2 \mathrm{~V}$. This was done to allow a longer battery autonomy, leading to longer measurement intervals. This had a negative effect because it reduced the load cell sensitivity. The calibration procedure relates the output voltage of each ring load cell with kiloponds, $\mathrm{kp}$, of compression. The term kilopond replaces kilogram-force, kgf, that was used before 1992. Fifty different compression force values were applied to the calibration set. Figure 5 shows the calibration results for one of the prototypes. The obtained characteristic is linear, with an $\mathrm{R}^{2}$ better than $99 \%$ for a straight line. This procedure was repeated after six months in order to evaluate the long-term stability of the prototypes. The calculated slopes, including the gain of the instrumentation amplifiers, showed a total error of $5 \%$ in the worst case. The nonlinearity obtained was $1.73 \%$ in the worst case.

The first prototypes used 24-bit sigma-delta converters. The digital signals were transmitted to a PC by a conventional serial interface at $38,400 \mathrm{bps}$. Further development included electronics inside the load cells. Low power $2.4 \mathrm{GHz}$ radio transceivers were included to send the compression force data to a PC over the air. To complete the system, $\mathrm{C}++$ software was developed to show and store the compression forces obtained by the load cell.

\section{Application of the 3-ring Spherical Load Cell}

Several different experiments were performed using the 3-ring load cell during the post-harvest processing of apples and oranges [1]. Maximum compressions of up to $18 \mathrm{kp}$ were found and repeated in a horticultural laboratory to investigate the real damage they produced on the fruits. The sphere was also placed in different positions inside the selection 


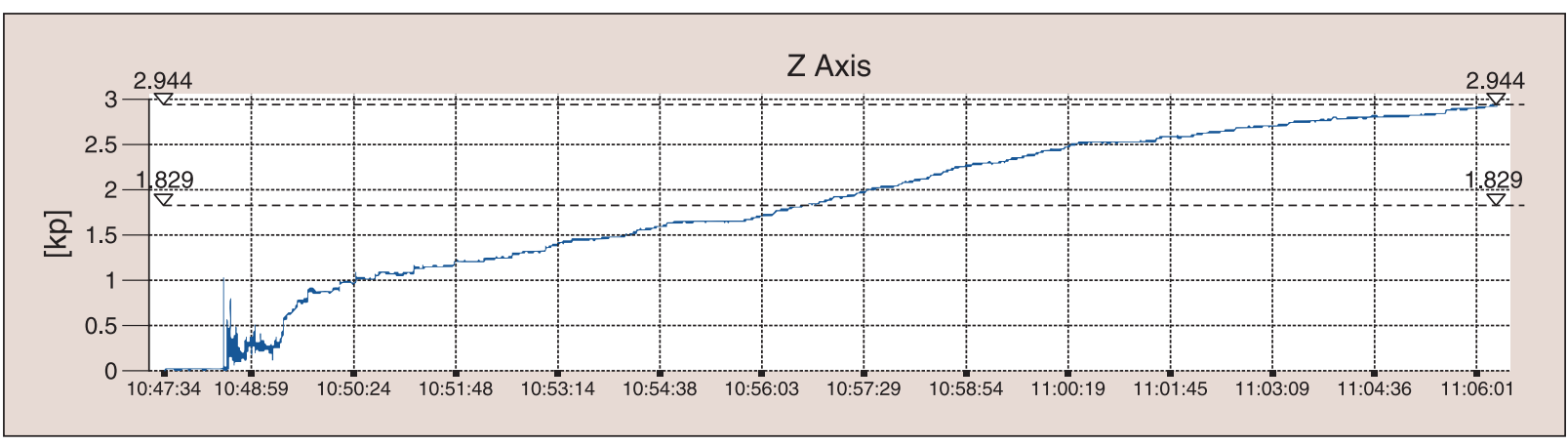

Fig. 6. Plotted data during a silo filling procedure.

machinery. Experiments were also conducted by placing the sphere inside the transportation boxes. They revealed strong compressions and impacts especially during pallet lifting and transportation. Figure 6 shows a graph of the compression on fruit during a silo filling experiment. During the first minutes of filling the silo, the graph shows individual impacts from the fruit. After the formation of layers of fruit, the load cell receives constant compression. This method of using spherical load cells to measure compression and create an impact analysis on fruits is very useful in developing better methods of handling agricultural products that are compressible in post-harvest agricultural activities.

For instance, as a result of one of the experiment's results of data collection and analysis of compression forces experienced by fruit in transportation boxes, the producer of the boxes decided to change its design in order to minimize compression forces.

\section{Summary}

We presented some of the theory of load cells, and a novel application of a three-ring spherical load cell in the construction of a versatile measurement system. Internal and external curvature of the sphere was a problem as it caused a Wheatstone bridge imbalance which was corrected by auxiliary parallel trimming resistance and software compensation. $\mathrm{C}++$ software was developed to show and store the compression forces obtained by the load cell. The versatility of the load cell system we designed permitted us to obtain data in different ways in the application of post-harvest processing of apples and oranges. Future work activities include the development of a new device employing wireless sensor technology to establish cooperative communication among the devices. This will permit more realistic experiments when the devices will be deployed as real fruit in a post-harvest system.

\section{References}

[1] I. Muller, R.M. de Brito and R.J. Bender, “Instrumented Sphere for Compression Analysis", in Proc. IEEE IIMTC 2008, 2008.

[2] "Strain gage: Theoretical Background", efunda, Inc., [Online] Available: fttp:/ / efunda.com/designstandards/sensors/strain_ gages/strain_gage_theory.cfm

[3] J.W. Dally, W.F. Riley, and K.G. McConnell, Instrumentation for Engineering Measurements, New York: John Wiley, 1993.
[4] J. Fraden, Handbook of Modern Sensors, 3rd Ed. Springer AIP Press, 2004.

[5] E.O. Doebelin, Measurement Systems Application and Design, New York: McGraw-Hill, 1990.

[6] R.A. Meyer, A.J. Kempaynen, and D.J. Olson, Multi-axis load cell body. U.S Patent Number 6,769,312,B2, Aug. 2004.

Ivan Muller (ivan.muller@ufrgs.br) received a B.S. degree in electrical engineering in 2003 and the MS Degree in 2008 from the Federal University of Rio Grande do Sul, Brazil (UFRGS). Currently, he is working towards a D.Sc. degree in automation systems. His main research interests are electronic instrumentation and measurement systems, wireless sensors network and power electronics. He is an IEEE student member.

Renato M. de Brito received a B.S. degree in electrical engineering in 1973 and the M.Sc. degree in 1994 from the Federal University of Rio Grande do Sul, Brazil (UFRGS) where he taught for thirty years. Today he is an invited professor and his main interests are electronic instrumentation, measurement and data acquisition systems and applied engineering systems.

Carlos E. Pereira received a B.S. degree in electrical engineering in 1987 and the MS degree in computer science in 1990 from the Federal University of Rio Grande do Sul, Brazil (UFRGS). He received the Dr.-Ing. Degree in electrical engineering from the University of Stuttgart, Germany in 1995. Currently he is professor at UFRGS and his main research interests are distributed real-time embedded systems, industrial automation applications and communication protocols.

Valner J. Brusamarello received a B.S. Degree in electrical engineering in 1992 and the MS Degree in Material Science in 1996 from the Federal University of Rio Grande do Sul, Brazil (UFRGS). He obtained the D.Sc. Degree in Material Science from Federal University of Santa Catarina, Brazil (UFSC) in 2000. Currently he is professor at UFRGS and his main research interests are electronic instrumentation, measurement and data acquisition systems and applied engineering systems. He is an IEEE member. 